\title{
Mobility Management Scheme Based On Identifier/Locator Separation in Mobile IP Environment for Future Internet
}

\author{
Thong Huynh ${ }^{\dagger}$, Won-Joo Hwang ${ }^{+\dagger}$
}

\begin{abstract}
One of the major issues in current internet architecture is that it was not designed to support the user mobility. In order to overcome this restriction, in this paper, we propose an identifier/locator separation architecture which contain the overlay mapping system to store the identifier-to-locator mapping record. In addition, we design the mobility management scheme base on Identifier/ Locator separation above for Furture Internet architecture. We then devise the analysis model to evaluate the signaling cost of our scheme. By conducting the simulation. we show that our scheme can operate with lower signaling cost than other schemes.
\end{abstract}

Key words: Future Internet, Identifier/locator Separation, Mapping System, and Mobility Management

\section{INTRODUCTION}

Since recent year, Future Internet has received a great deal of attention due to the wide availability of wireless services and mobile devices. Various technologies base on Future Internet such as 3G/ $4 \mathrm{G}$ cellular system, LTE system were designed to provide the hight-data-rate mobile services with quality of service (QoS) requirement. There are many active researches focusing on the designing the Future Internet architecture in order to overcome the limitation of the current internet such as FIND [1], GENI [2] in United States; AKARI [3] in Japan, FIF [4] in Korea. Such extensive works

※ Corresponding Author: Won-Joo Hwang, Address: 621-749, Obangdong, Gimhae, Gyeongnam, Korea, TEL: +82-55-320-3847, FAX : +82-55-322-6275, E-mail : chwang @inje.ac.kr

Receipt date: Nov. 23, 2012, Revision date: Dec. 14, 2012 Approval date: Dec. 21, 2012

${ }^{+}$Department of Information and Communication Engineering, HSV-TRC, Inje University

(E-mail: huythongtc@gmail.com)

${ }^{++}$Department of Information and Communication Engineering, HSV-TRC, Inje University

※ This research was supported by Basic Science Research Program through the National Research Foundation of Korea (NRF) funded by the Ministry of Education, Science and Technology (2011-0024888). on the Future Internet address the problem of the routing/addressing architecture, mobility management, security, etc.. Particularly, mobility management is one of the key issue in Future Internet in order to support uninterrupted service during the movement of mobile node between different location.

However, one of the major issues in current internet architecture is that it was not designed to support the user mobility. This is because the current $\mathrm{IP}$ address are used both to identify the interface of a host which is connected to Internet and to all of the application layer, IP address identify a host and it is used in the application and transport layer simultaneously [5]. From the view of the network layer, IP address represents the current topological location of the host and forward packet to the host. There is no independent identifier to represent the host.

When the host moves to a new location or network, it will obtain the new IP address, all the seasons from the application, transport layer related with the previous address are broken and cannot naturally restored. This architecture makes it difficult to provide the new requirements of supporting user mobility in the Future Internet. The other rea- 
son for the Internet does not support effectively mobility is the limitation of the Domain Name System(DNS). The current DNS server can not map the name to the locator quickly when the host change their IP address due to mobility. DNS alone is not sufficient for the Future Internet.

To solve the above mentioned problem, many studies on Identifier/Locator Separation architecture such as HIP [6], LIPS [7], MIP [8], etc. have been carried out to support the incremental deployment of the Identifier/Locator Separation in the current Internet. However, most of them required many signalling traffic in order to update the location of the user. Fewer signalling traffic will lead to more data traffic while more signalling traffic will result in less data traffic Hence, the signalling traffic must be considered in designing the mobility management scheme. In this paper, we apply the Identifier/Locator Separation to decouple the identifier from the location. It has been shown that the Identifier/Locator Separation is a basic component of the Future Internet architecture. It will allow user to move from one location to another location without any connection disruption. Then a novel mobility management scheme is designed in this Identifier/Locator Separation context.

Our work can be summarized as follow:

- By introducing the mapping server, we separate the identifier from the locator. Each user can move to other location without any change the host name at the application layer. Then we propose the mobility management scheme in order to keep the mapping between the identifier and its dynamic changing IP address due to the operation of the Identifier/Locator Separation above (Section 2).

- We establish analytical model and formulate the signalling cost under the distribution function (Section 3). The result show that our scheme can reduce efficient the signalling cost compared which the MIP and LIDS schemes [9] (Section $4)$.

\section{MOBILITY MANAGEMENT DESIGN}

In this section, we propose an ID/Locator separation architecture and design a mobility management based on this architecture.

\subsection{ID/Locator Architecture}

The proposed architecture is illustrated in Fig. 1, which is composed three layer: management layer, core layer, access layer. The management layer consists of a set of the mapping server (MS) that manage identifier-to-locator mapping entries and resolve the mapping request message. The core layer is actually the current Internet's backbone which use the locator to transmit the packet. The core layer uses the IP based routing mechanism, thus it can adapt any new routing based on IP in the future. The user layer consists of topological physical network. It is divided into a number of organization areas (OA). Each OA is managed by the edge router (ER). The ER in this layer bridges the access layer and the core layer. The ER includes the functionality such as assigning the locator to the user, mapping the ID and locator for

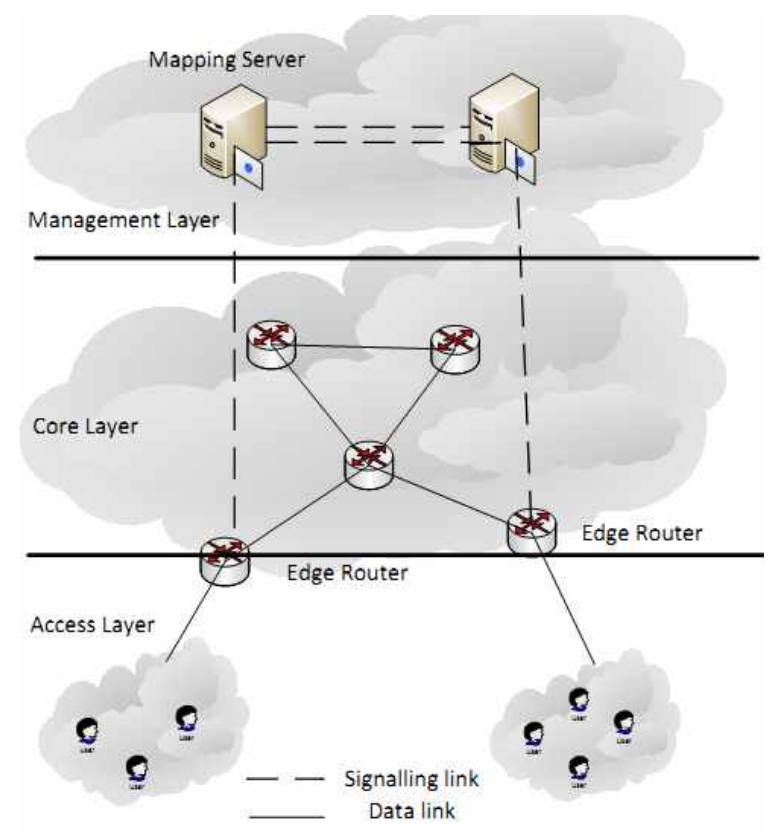

Fig. 1. Network architecture for mobility management. 
incoming and outgoing packets.

\subsection{Motivate Example}

We start with an example consisting one user moving in a region as shown in Fig. 2. Suppose that the service provider network is 2.x.x.x with 2.3.x.x and 2.4.x.x sub networks assigned to different physical region. In this example, the Domain Name Service (DNS) server allocates a home address for the user : 2.2.1.1 as user's identity. When the user moving into a new subnet region, the ER will assign the IP address (physical address) to the user. In this figure when the user move to the subnet 2.3.x.x, the ER will assign 2.3.1.1 as the address for this user. This mapping can be stored at the mapping server. Notice that, only the MS knows the mapping between the user identity (2.2.1.1) and the location (2.3.1.1). The user's identity is used regardless of the mobility of user. When the user move to 2.4.x.x sub-net, the location is updated to 2.4.1.1, not its identity.

\subsection{Mobility Management Scheme}

We divided the network into several OAs. In this scenarios, the mobile user 1 (MU1) move into a new $\mathrm{OA}$ and it want to transmit data to the mobile user 2 (MU2) through their edge router: ER1 and

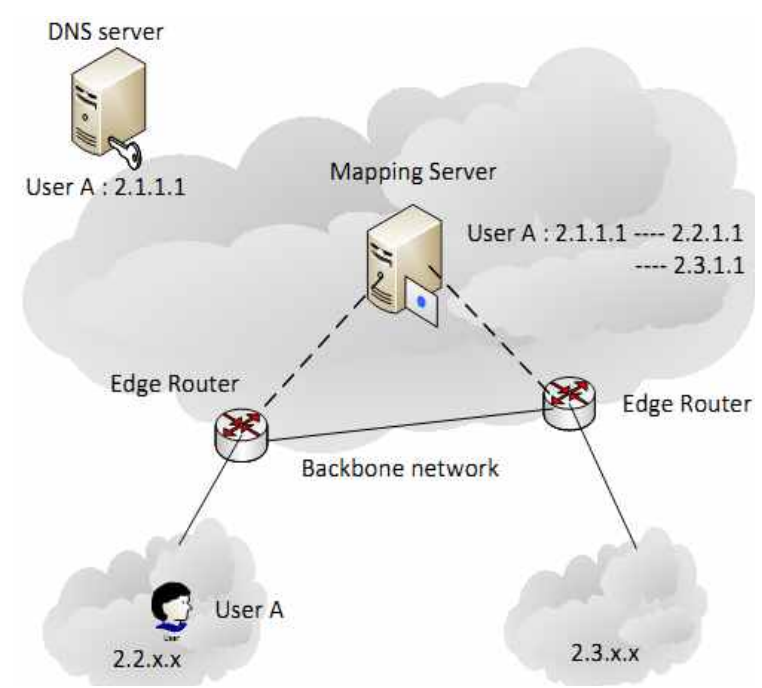

Fig. 2. Motivate example.
ER2.

Thus, the mobility management process is executed in order to avoid the transmission interrupt. The mobility process is illustrated in Fig. 3. First of all, MU1 sent the access message to its ER, ER1, to inform its identity. After receiving the message, the ER1 send a mapping registration request including the MU1's identity and locator to the MS. The MS adds the mapping entry and sent a mapping report message to the ER2 and ER1 to update the mapping. When the RE2 receives this report message, it will update the cache of MU1 's locator mapping and transmit the following message according to the new mapping. Then the communication is continuing.

In our architecture, mobile users have to update their location to the mapping server after moving to a new OA. The location management is achieved through the use of the DNS server and the others

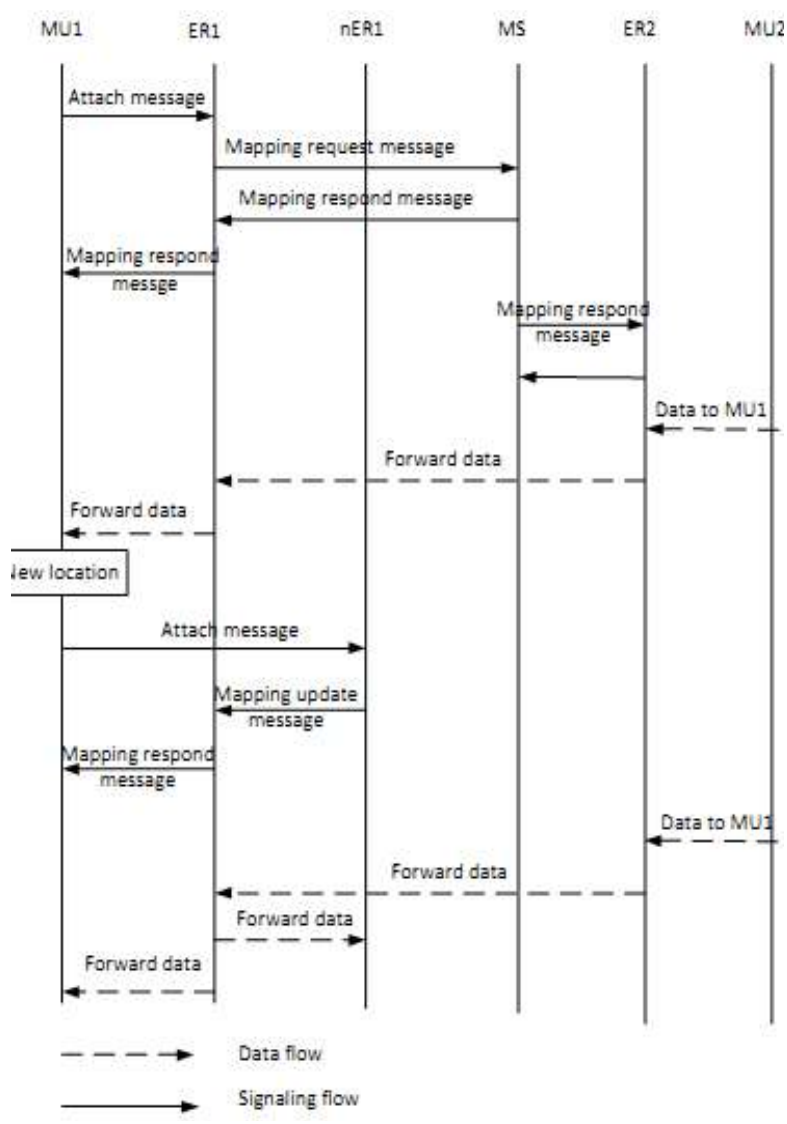

Fig. 3. Message flows of the mobility managemetn scheme. 
mapping servers which are responsible for the node's location storage. Our proposed architecture has separated the name resolution from the locator update mechanism. Whenever a mobile user moves into other OA, the user updates its new IP address to the MA server in order to be reachable after mobility event while keeping the identity.

In order to reduce the update message to the MS, when the MU1 moves into the new OA with new edge router nER1, the MU1 can send an access message to the ER1 and the ER1 only send mapping update message to nER1. The nER1 send the respond message and establish the tunnel with the ER1 in order to forward message to MU1. All the message from MU2 will pass through ER1 and then ER1 will forward this message to nER1 by tunnel. In this way, the mapping information in ER2 and MS do not change. so it will be reduce significantly signalling cost. However, this scheme will increase the delay of the system due to the message passing through tunnel. To overcame this issue, we limit the number of the router in tunnel by $\mathrm{K}$. When the number of the edge router in tunnel excess $\mathrm{K}$, the ER sent the mapping information to MS and update the new mapping.

\subsection{Mapping Table Design}

In order to store the mapping record of the MU, we design the mapping table in the MS. The mapping table as shown in Table 1 contains four sections. "ID" is the identifier of MU. "Locator" is the IP address of the MS for locating node in network topology. "FLAG" indicates the status of the "Locator". If the value of the FLAG is DEAD, the current locator cannot be used any more. On other hand, the value of the FLAG is ALIVE, the locator can be use. The function of "TIMER" is to manage the MU's mapping entry. When "TIMER" exceeds a predetermined threshold, the MS sent the keep

Table 1. The mapping table in mapping server

\begin{tabular}{|c|c|c|c|}
\hline ID & LOCATOR & FLAG & TIMER \\
\hline
\end{tabular}

alive message to the MU. If the MS receive respond message from the MU, then the MS reset TIMER to zero.

\section{PERFORMANCE ANALYSIS}

In this section, we develop an analysis model to study the signalling cost in our scheme. We define the following notations for our analysis.

$c_{m e}$ : The transmission cost between mobile user and edge router.

$c_{r s}$ : The transmission cost between edge router and mapping server.

$c_{m s}$ : The transmission cost between mobile user and mapping server.

$c_{e e}$ : The transmission cost between edge router

$a_{m}$ : The processing cost at mobile user

$a_{e}$ : The processing cost at edge router

$a_{s}$ : The processing cost at mapping server

For performance analysis, we consider the analysis model similar to [10]. We assume that there are $\mathrm{N}$ subnets, and $\mathrm{k}$ subnets constitute an OA. Each MU can move from one subnet to the other subnet with equal probability $\frac{1}{N-1}$ according to a Markovian random walk. We call the action each MU moving out of a subnet "a step". We define a random variable $\mathrm{M}$ so that each MU move out the OA at step M. Then according to [10], the probability that the MU move out of the OA at the step $m$ is:

$$
p_{m}=\frac{N-k}{N-1}\left(\frac{k-1}{N-1}\right)^{m-2}
$$

Then the expectation of $\mathrm{M}$ is:

$$
E[M]=\sum_{m=2}^{\infty} m p_{m}=1+\frac{N-1}{N-k} .
$$

In our scheme, when the user moves between the OA $\mathrm{K}$ times, then the location update cost is shown as follow:

$$
\begin{aligned}
& U=\underbrace{1}_{2 c_{m e}+2 c_{e s}+2 c_{e s}+a_{n}+4 a_{e}+2 a_{s}} \\
& +\underbrace{(K-1)\left(2 c_{m e}+c_{e e}\right)}_{2}
\end{aligned}
$$


where term 1 indicate the cost of initial mapping update message to MS. The term 2 show the local update between each $\mathrm{ER}$ in order to establish tunnel.

To compare and analyse the performance of our scheme, we employed the MIP [8], LIDS [9] schemes in the above analysis model. The location update costs of those schemes are delivered as follow:

$$
\begin{aligned}
& U_{L I D S}=K\left(6 c_{r s}+c_{m e}+2 c_{e e}+5 a_{e}+2 a_{s}\right) \\
& U_{M I P}=K\left(4 c_{m e}+2 c_{m s}+3 a_{n}+2 a_{e}+a_{s}\right)
\end{aligned}
$$

In additional, we assume that the incoming calls to a portable are a Poisson process. Let $\beta(i)$ be the probability that a MU move across i subnets between two phone calls. Then, the average update cost of three schemes is:

$$
\begin{aligned}
& C=\sum_{i=0}^{\infty}\left(\frac{i}{E[M]}\right) \frac{U}{K} \beta(i) \\
& C_{L I D S}=\sum_{i=0}^{\infty}\left(\frac{i}{E[M]}\right) \frac{U_{L I D S}}{K} \beta(i) \\
& C_{M I P}=\sum_{i=0}^{\infty}\left(\frac{i}{E[M]}\right) \frac{U_{M I P}}{K} \beta(i)
\end{aligned}
$$

Notice that, in three schemes above, $\frac{1}{E[M]}$ is average probability of moving out of $\mathrm{OA}$ at each step. Then, $\frac{i}{E[M]}$ is the average probability move out of OA during a call. We assume that the probability $\beta(i)$ of i moves between two income calls has a exponential distribution:

$$
\beta(i)=\frac{\rho}{(\rho+1)^{i+1}}
$$

where $\rho=\frac{\lambda_{c}}{\lambda_{m}}$ is the call-to-mobility ratio [11], [12] $\lambda_{c}$ is the call-arrival rate and $\lambda_{m}$ is the cross OA rate.

\section{PERFORMANCE EVALUATION}

Base on the analysis in section 3, in this section we give the analytical performance result of the total signalling cost.

Fig. 4 show the impact of the call-to-mobility

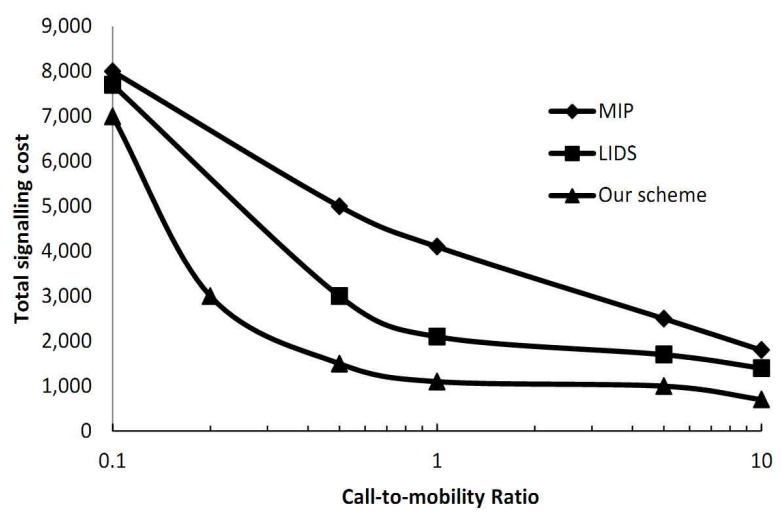

Fig. 4. Comparison of total signaling cost under variance of $\mathrm{CMR}$.

ratio to the signalling cost. For all mobility management schemes, it can be observed that the signalling cost of three schemes decrease as the CMR increase. This is because when the CMR is low, the mobility rate is high compared to the packet arrival rate. So the user must execute the location update more frequently. It will lead to the high signaling cost. In addition, the total signaling cost of our schemes is smaller than the one of MIP and LIDS. This is because our scheme establish a tunnel to forward the message to the user when user moves to a new location. It no need to inform new location to the mapping server for each movement of OA. thus our scheme avoids much signaling cost in the heavyweight wireless link. Therefore the low signaling cost can be achieved in our scheme.

Next, we consider the impact of the number of user to the signaling cost of our scheme. We vary

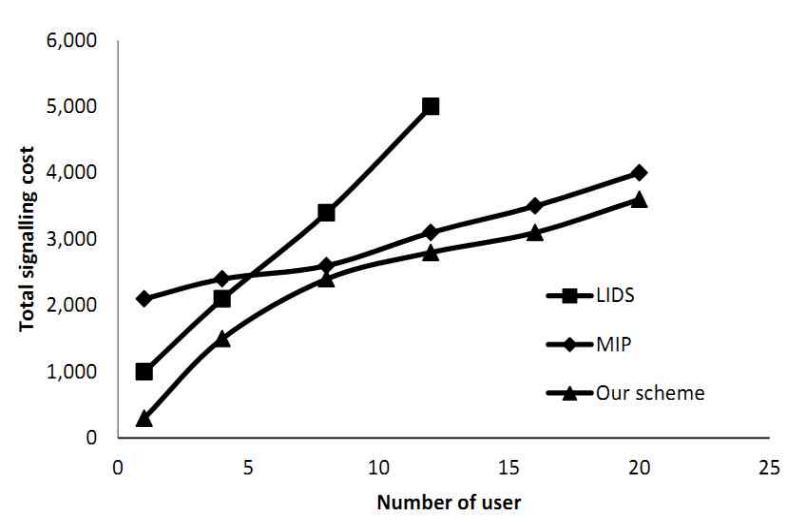

Fig. 5. Comparison of total signaling cost under variance of user. 
the number of user from 1 to 20. As shown in Fig. 5, the signalling cost increases as the number of user increase. This is because when the number of user increase, the new mapping is required more to be update the locator for users. It will lead to the cost is proportional the number of the user. Notice that, our scheme alway obtain the lower cost than two others policy regardless the number of user in the system.

Finally, we analyze the effect of the value $\mathrm{K}$, number of the router in tunnel, to the signaling cost of the system. The result is given in Fig. 6. As shown in this figure. we can observer that value $\mathrm{K}$ affect significantly to the total signaling cost. First, when the value $\mathrm{K}$ increases, the total signaling cost decreases due to the reduce the update times of user. However, when the value of $\mathrm{K}$ increases greater than a threshold value, the cost will increase. This is because a larger value of $\mathrm{K} \mathrm{re}^{-}$ quires a longer path to forward data to user. Thus, user need more time to transmit data to each other and affect to the average signaling cost of the system.

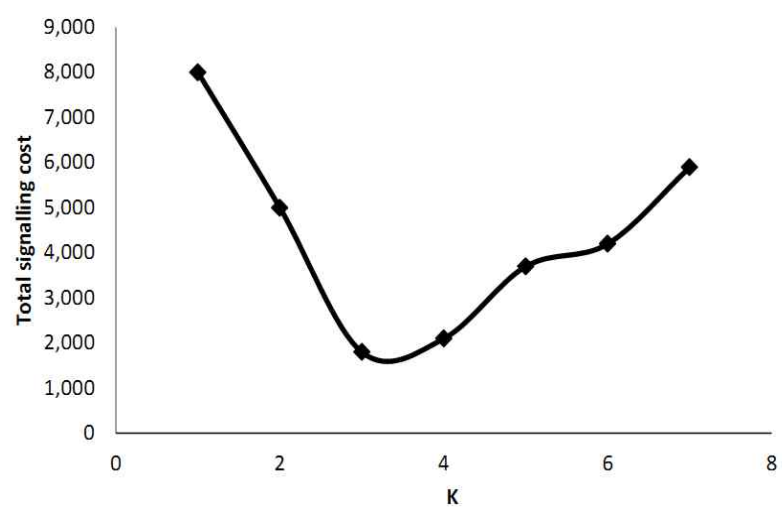

Fig. 6. The total signaling cost under variance of number of router in tunnel.

\section{CONCLUSION}

In Future Internet, user will be using device with highly mobile. Therefore, these technologies in Future Internet should support key future such as full support for user mobility. In this paper, we have designed the mobility management scheme base on the identifier/locator separation. We propose the model to analyze the performance signaling cost of this scheme. The simulation was conducted to show that our scheme has the signaling cost lower than other schemes.

\section{REFERENCES}

[1] FIND, http://www.nets-find.net, 1997.

[2] GENI, http://www.geni.net, 2007.

[3] AKARI, http://akari-project.nict.go.jp, 2007.

[4] FIF, http://fif.kr, 2006.

[5] H. Balakrishnan, K. Lakshminarayanan, Ratnasamy, and M. Walfish "A Layered Naming Architecture for the Internet," Proc. Applications, Technologies, Architectures, and Protocols for Computer Communications, pp. 343-352, 2004.

[6] R. Moskowitz, P. Nikander, P. Jokela, and T. Henderson "Host Identity Protocol," RFC 5201, pp. 5-102, 2008.

[7] D. Meyer, "The Locator Identifier Separation Protocol (LISP)," The Internet Protocol Journal, Vol. 11, No. 1, pp. 23-36, 2008.

[8] C. Perkins et al., "IP Mobility Support for IPv4," RFC 3220, Internet Engineering Task Force, pp. 3-98, 2002.

[9] Z. Yan, H. Zhou, H. Zhang, and S. Zhang, "A Novel Mobility Management Mechanism Based on an Efficient Locator/ID Separation Scheme," Proc. First International Conference on Future Information Networks, pp.11-16, 2009.

[10] J. Xie and I.F. Akyildiz , “A Distributed Dynamic Regional Locaion Management Scheme for Mobile IP," Proc. IEEE INFOCOM, pp. 1069-1078, 2002.

[11] R. Jain, Y.B. Lin, C. N. Lo, and S. Mohan, "A Caching Strategy to Reduce Network Impacts of PCS," IEEE Journal on Selected Areas in Communication, Vol. 12, No. 8, pp. 
1434-1444, 1994.

[12] R. Casaquite, and W. Hwang, "Design and Implementation of HomeTDMA: a TDMA Protocol for Home Networks," Journal of The Korea Multimedia Society, Vol. 10, No. 12, pp. 786-798, Dec. 2007.

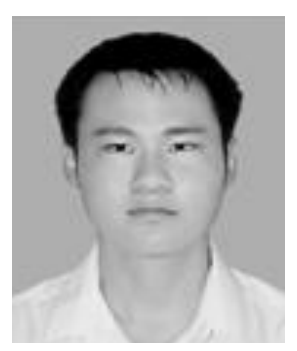

\section{Thong Huynh}

Received his bachelor's degree in Information and Communication System from HoChiMinh University of Technology, Vietnam in 2004 and M.E. from Department of Information and Communications Engineering, Inje University, Korea. $\mathrm{He}$ is currently $\mathrm{PhD}$ candidate in the same department. His research interests are cross-layer design and optimal control in wireless networks.

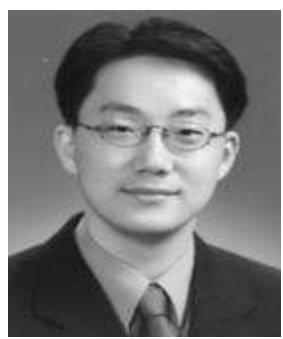

Won-Joo Hwang

Received his Ph.D Degree from Osaka University Japan in 2002. He received his bachelor's degree and M.S. degree in Computer Engineering from Pusan National University, Pusan, $\mathrm{Re}^{-}$ public of Korea, in 1998 and 2000. Since September 2002, he has been an assistant professor at Inje University, Gyeongnam, Republic of Korea. Currently, he is the director of the Computer Networks Laboratory of the same school. His research interest is in network optimization and ubiquitous sensor networks. 